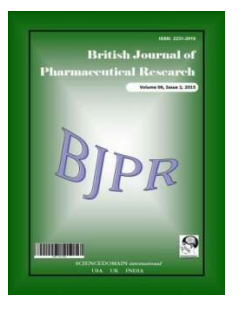

British Journal of Pharmaceutical Research

14(5): 1-12, 2016; Article no.BJPR.31788

ISSN: 2231-2919, NLM ID: 101631759

SCIENCEDOMAIN international

www.sciencedomain.org

\title{
Potential for a Beneficial Effect of Theophylline on Extended Acute Inflammation: Review
}

\author{
S. C. Allen ${ }^{1,2^{\star}}$, G. T. C. Wong ${ }^{3}$, M. Vassallo ${ }^{1,2}$ and J. S. K. Kwan ${ }^{3}$ \\ ${ }^{1}$ Centre for Postgraduate Medical Research and Education, Bournemouth University, Dorset, UK. \\ ${ }^{2}$ The Royal Bournemouth Hospital, Dorset, UK. \\ ${ }^{3}$ Faculty of Medicine, University of Hong Kong, Hong Kong SAR, China.
}

Authors' contributions

This work was carried out by collaboration between all the authors. Author SCA performed the literature search, formulated the hypothesis and wrote the first and final drafts of the paper. Author GTCW contributed to the hypothesis, clarified the patterns of inflammation and commented on the drafts. Author MV contributed to the hypothesis and reviewed the first draft. Author JSKK contributed to the hypothesis and reviewed the first and final drafts. All authors read and approved the final manuscript.

\section{Article Information}

DOI: 10.9734/BJPR/2016/31788

Editor(s):

(1) Salvatore Chirumbolo, Clinical Biochemist, Department of Medicine, University of Verona, Italy. Reviewers:

(1) Alcibey Alvarado González, Clínica de Diagnóstico Médico, San José, Costa Rica. (2) Tânia Silvia Fröde, Federal University of Santa Catarina, Brazil. Complete Peer review History: http://www.sciencedomain.org/review-history/17912

Mini-review Article

Received $25^{\text {th }}$ January 2017

Accepted $13^{\text {th }}$ February 2017

Published 21 ${ }^{\text {st }}$ February 2017

\section{ABSTRACT}

Inflammation is often prolonged after sepsis, trauma and other acute pro-inflammatory events, particularly above the age of 80 years. Exposure to inflammation of inappropriate amplitude or duration is associated with a higher risk of delirium, anorexia, lethargy, low mood, weakness and other markers of frailty, and with less favourable clinical outcomes. Theophylline has been shown in vitro and in vivo to have an anti-inflammatory effect, probably mediated through induction of histone deacetylase-dependent gene switching in immune competent cells. This is mainly characterized by a reduction in the production and release of TNF $\alpha$, IL-1 $\beta$ and IL- 6 , a consequent fall in CRP and increase in IL-10, and a shift of immune cell phenotypes to the anti-inflammatory mode. This effect occurs at theophylline concentrations in the 5-10 mg/L range, which is below the broncho-dilator range $(10-15 \mathrm{mg} / \mathrm{L})$ and carries a relatively low risk of toxicity. We hypothesize that low-dose theophylline treatment given to elderly subjects with acute inflammation, for example due 
to pneumonia, septicaemia or trauma, will alter the balance of their inflammatory status from an inappropriately extended pro-inflammatory pattern toward a more normalized baseline pattern and thereby reduce the risk of adverse clinical outcomes.

Keywords: Systemic inflammation; old age; frailty; cytokines; theophylline; anti-inflammatory drugs.

\section{INTRODUCTION}

A substantial body of evidence indicates the involvement of systemic inflammation in the pathogenesis of several aspects of the frailty syndrome [1,2] most frequently encountered in old age. This is a complex field that spans clinical medicine, immunology, pathology and biological chemistry, and there is a fragmentary understanding of the regulatory mechanisms involved, the range of normative states and the variations over time. However, for the purposes of this paper the chemical and cellular systemic inflammatory status of an individual can be broadly envisaged in the following categories:

1. Normal: This is the usual condition of healthy individuals between episodes of acute inflammation. Various cytokines and other bio-chemicals are found in peripheral blood in an approximately steady state at low concentration, or are undetectable, and immune competent cells express a mainly surveillance phenotype.

2. Chronic low-amplitude inflammation: This state is frequently encountered in older people, even those who appear to be healthy. It is also associated with a wide range of chronic pro-inflammatory conditions that have a high prevalence in elderly people, including chronic obstructive pulmonary disease (COPD), osteoarthritis, type 2 diabetes, obesity and a sedentary lifestyle [3-8].

3. Acute inflammation with appropriate resolution: This is the normally observed response to events such as infection or trauma in healthy individuals. Typically, there is a rise in circulating proinflammatory bio-chemicals and cells, then an anti-inflammatory surge, followed by a rapid return to pattern 1 after the initial stimulus has been eliminated.

4. Acute inflammation with inappropriate resolution: This is a less well recognized but not uncommon response to ostensibly ordinary infections, such as pneumonia, urinary tract infections, Gram-negative septicaemia and cellulitis, or other physiological insults such as accidental or surgical trauma. In older patients, the proinflammatory phase in such circumstances is often prolonged $[2,9,10]$ and is associated with a number of clinically important consequences such as delirium, lethargy, low mood, autonomic dysfunction, reduced appetite, weakness and increased dependency. There is a slow, and sometimes incomplete, return to baseline (either pattern 1 or 2). Geriatricians and other clinicians involved with the care of elderly people are becoming increasingly familiar with this phenomenon and the adverse effect it can have on a range of outcomes for their patients.

5. Overwhelming and dysregulated inflammation: This is a comparatively rare but torrential and frequently fatal acute disorder of innate immunity leading to severe sustained systemic inflammation in some patients with sepsis, burns or trauma, and is usually encountered in the critical care setting [11]. It can occur at any age but tends to have a very poor prognosis in older patients.

As part of this review paper, we will present a hypothesis that contends that the outcomes from pattern 4, usually encountered in elderly patients and sometimes in younger people with chronic inflammatory conditions, could be improved by using the methyl-xanthine drug theophylline to modify the balance of pro-inflammatory and antiinflammatory entities (mostly cytokines) closer to that observed in pattern 3 so that the deleterious effects of extended acute systemic inflammation are measurably reduced.

\section{BACKGROUND}

\subsection{Innate Immunity}

The cells and cytokines of the innate immune system are the first responders to infection and other forms of tissue damage. This ancient and evolutionarily robust defence mechanism is represented in some form in almost all multicellular animals. It operates in advance of and modulates adaptive immunity in individual organisms and almost certainly evolved much earlier [12]. The chemistry and cell biology of innate immunity is complex and incompletely understood, and it is not the purpose of this 
paper to attempt an extensive review of that topic. However, in support of our hypothesis it is necessary to describe some relevant features of the system. Though some empirical findings have been equivocal or even contradictory there is a sufficient amount of consistent research to be able to form a useful understanding of a number of key processes that are informative within the context of this paper. Taking pneumococcal infection as an example, in individuals with or without adaptive antibody immunity, pneumococcal antigens are initially presented to tissue macrophages and circulating monocytes both directly and as complexes bound to C-reactive protein (CRP), serum amyloid A (SAA) and toll-like proteins. In response, monocytes of pro-inflammatory phenotype release pro-inflammatory cytokines of the interleukin-1 (IL-1) family, interleukin-6 (IL-6) and tumour necrosis factor- $\alpha$ (TNFa), probably within 1-3 hours as shown by in vitro studies $[13,14]$. There is some evidence that this process is delayed in older patients, based on studies in a clinical setting [15], but the pathophysiological and clinical significance of that finding is not clear. By processes for which there is only a partial understanding those cytokines, other less extensively studied cytokines, and other biochemicals involved in innate immunity such as interferons and complement, stimulate a number of responses by a wide range of cell types that contribute to an effective elimination of the infection. The range of these pro-inflammatory effects also includes increased production of CRP and SAA by hepatocytes, increased endothelial permeability, reduced protein synthesis and increased interleukin-6 (IL-6) release by various cells including monocytes, myocytes and adipocytes, reduced albumin synthesis, switching of neutrophils to a proinflammatory phenotype that includes further release of pro-inflammatory cytokines including TNFa, down-regulation of autonomic cardiovascular reflexes, and context-variable effects on synthetic functions by other tissues and metabolic pathways including glucocorticoid and catecholamine production $[1,16]$. Lethargy and an urge to rest, sometimes referred to as sickness behaviour, are probably at least partly due to cytokine-mediated alterations in central nervous system function. There is also circumstantial evidence that pro-inflammatory cytokines directly predispose to delirium [17], though the mechanism is not clear and other mechanisms, such as cerebral capillary microcoagulation, might be at play. There is a complex effect on macrophages leading initially to further production of IL-1 $\beta$, TNFa and the antiinflammatory cytokines interleukin-4 (IL-4) and IL-1 receptor antagonist (IL-1ra). Antigenstimulated monocytes are also self-stimulated to release TNF and other cytokines by an autocrine mechanism and, through an influence on $\mathrm{T}$ helper cell function, lead to rapid production and release of interferon-y (IF-y), which in turn contributes to the response to infection by a range of interactions with cells of the innate and adaptive immune systems [16]. The essential concepts of this process are summarized in simplified form in Fig. 1. In the early and mature phases of this pro-inflammatory response the primary purposes of eliminating infection, minimising damage and the survival of the individual are being served (pattern 3). Once infection has been eliminated counter-regulatory mechanisms switch the chemokine and cellular milieu of the innate immune system to an antiinflammatory phase characterized by a rapid fall in IL-1 $\beta$, TNF $\alpha$, other pro-inflammatory cytokines and CRP, a transient rise in blood levels of antiinflammatory cytokines such as IL-4, interleukin10 (IL-10) and IL-1ra, and a return of immune cell phenotypes to mainly anti-inflammatory and surveillance mode (pattern 1) [18].

\subsection{Innate Immune Responses in Old Age}

The control of this process of switching from a defensive pro-inflammatory state back to baseline is poorly understood and very complex but appears to take longer and to be less clearly complete in many elderly individuals $[9,10,19]$, such that the persistence of an inflamed state beyond the time of active infection starts to have deleterious effects. Consequently, in many elderly people the biochemical and clinical proinflammatory response to acute infection does not resolve as quickly as it does in younger adults (pattern 4). The rises in IL-1 $\beta$, TNF $\alpha$, IL-6 and CRP persist longer, and the rise in antiinflammatory IL-10 is delayed and also prolonged [20]. This effect is reflected in the observed time course of clinical recovery, and has been quantified in research on humans with pneumococcal infection and volunteers exposed to endotoxin that showed an approximate 2-fold longer time for biochemical markers of inflammation, such as TNF $\alpha$ and IL-1 $\beta$, to return to baseline in older individuals $[9,10]$. That research was conducted in healthy elderly people but evidence indicates that frailer elderly patients, with higher levels of background inflammation (pattern 2), have an even more extended period of persisting inflammation after 
acute infections [12]. The clinical correlates for that phenomenon include persisting anorexia, low mood and lethargy, incomplete resolution of delirium and CRP levels that do not return fully to the normal range. This suggests an impairment of regulatory anti-inflammatory function in old age that is a likely substrate for slower clinical recovery resulting from an extended exposure to the clinically negative effects of certain proinflammatory cytokines, particularly TNFa and IL$1 \beta$.

\subsection{Chronic Inflammation in Old Age}

To set the background further it is important to outline the observed inflammatory state associated with old age. Baseline biochemical markers of inflammation, such as IL-1 $\beta$, TNFa and CRP are often chronically raised 2- to 4- fold in the peripheral blood of elderly people (pattern 2 ), particularly those above the age of 80 years, compared with younger adults $[8,21]$. The reasons for this are not entirely clear, but in some individuals, it can be explained by the presence of a clinically obvious chronic inflammatory pathology such as COPD or rheumatoid arthritis [6]. Similar levels of TNFa and CRP are often found in elderly people with other disorders that are known to have a proinflammatory effect, such as central obesity, atherosclerosis or type 2 diabetes $[4,22]$. Several other conditions, common in old age, have been shown to be associated with chronically raised pro-inflammatory markers, though the causeand-effect relationship is less clear. These include Alzheimer's disease, chronic kidney disease, osteoarthritis and physical inactivity [23-25]. Some studies have described elevated baseline CRP and pro-inflammatory cytokines in elderly people with no apparent underlying chronic disease [8], thus supporting the contention that old age is in itself a proinflammatory condition. Of course, an alternative interpretation of that observation is that older individuals have an appropriate augmentation of innate immunity to counteract the neoplastic and auto-immune tendencies of the senescent phenotype.

In its chronic state, persisting low-amplitude inflammation is associated with a number of important adverse outcomes, including increased all-cause mortality, reduced muscle strength, impaired ability to carry out day-to-day essential tasks and lower self-reported health status [26]. Further, it appears that chronic disease progression is at least partly a consequence of chronic inflammation and not simply a manifestation of it, so there is a complex interaction between cause and effect that seems to be self-sustaining and deleterious [27]. The best example of this is perhaps the endothelial inflammation that occurs in atheromatous vascular disease, which can be envisaged as a destructive endo-vasculitis both resulting from and stimulating an enhanced systemic proinflammatory baseline state [28]. This chronic slightly inflamed state, with the associated and probably consequent increase in vulnerability to an overtly frail clinical condition, leads to a consideration in the following paragraph of the resulting deterioration that occurs when an acute infection or trauma is superimposed (Fig. 1).

\subsection{Probable Mechanisms for the Deleterious Effect of an Extended Acute Pro-inflammatory State}

It is necessary to consider the possible mechanisms whereby TNF $\alpha, I L-1 \beta, I L-6$ and other inflammatory bio-chemicals contribute to adverse clinical phenomena. It must be emphasized that our description of this is to some extent speculative but it is nevertheless the starting point for the hypothesis described below. To give an important example from the clinical perspective we will consider inflammationassociated neuronal dysfunction. There is evidence that pro-inflammatory over-activity and inadequate or incomplete anti-inflammatory activity contribute to delirium, low mood and sickness behaviour all of which are of considerable importance in the clinical setting [29]. Autonomic dysfunction is another probable consequence, though interpretation is to some extent confounded by the immuno-regulatory role of autonomic neuroendocrine pathways. Receptors for several cytokines are present on neurons and glial cells, including TNFa, IL-1 $\beta$, IL6 and IL-1ra, and transport systems have been demonstrated that enable a number of cytokines to enter the cerebro-spinal fluid and brain interstitial fluid, including IL-1 $\beta$, TNF $\alpha, I L-6$ and IL-1ra [30-33]. In animal experiments, it has been shown that measurable changes of behaviour, such as anorexia, hiding and sleepiness, are induced by exposure to IL-1 $\beta$ and TNFa [34], which are thought to act via hypothalamic neurons. The role of cytokines in neuronal function is complex and appears to be both deleterious and protective depending on the metabolic and patho-physiological context [34-36]. Recent in vitro evidence suggests that the influence of cytokines on brain function is to a 
considerable extent mediated by receptors on various cell types in the blood-brain barrier, leading to changes in permeability and the secondary production of pro-inflammatory cytokines beyond the barrier by glial cells [35]. In any case, the close temporal association of IL$1 \beta$, IL- 6 and TNFa elevation in peripheral blood with neuronal dysfunction during and after infection in old age is strongly suggestive that those cytokines are either directly or indirectly involved.

Though some of the adverse effects of IL-1 $1 \beta$ and TNFa are due to direct receptor-mediated changes in cell metabolism, there is evidence of widespread influences of an enhanced proinflammatory state on other metabolic pathways and cell functions that are less clearly direct. These include changes in insulin sensitivity, increased catecholamine and glucocorticoid secretion, increased production of reactive oxygen species in endothelium, augmented production of prostaglandins and stimulation of IF- $y$ release. Important indirect mechanisms include complement-mediated micro-coagulation as a consequence of a pro-inflammatory state [37]. The biological consequences of these changes are incompletely understood but some of the better described phenomena are of considerable importance in clinical geriatric practice, particularly the central and peripheral neuronal effects described above, reduced muscle protein synthesis, impaired myocyte function and increased capillary permeability.

\subsection{The Anti-inflammatory Properties of Theophylline}

Theophylline, a methyl-xanthine drug, has been used as a phosphodiesterase inhibitor bronchodilator for the treatment of asthma and COPD for many years. Its efficacy in that clinical context is limited by the need to use plasma concentrations in a therapeutic range (10-15 $\mathrm{mg} / \mathrm{L})$ that is close to the toxic range (>20 mg/L) and its clinical utility has been diminished by the superior performance of beta-2 agonist and antimuscarinic bronchodilators. However, it was observed that certain favourable patient outcomes, such as walking performance, were reported in patients with COPD given theophylline treatment even when little or no measurable change occurred in spirometry indices of airways obstruction or arterial blood gas tensions, and the effect was also observed at low (<10 mg/L) plasma levels [38]. Further research demonstrated anti-inflammatory properties of theophylline locally on airways inflammation, systemically and in vitro [38,39]. Other methyl-xanthine drugs such as pentoxifylline have similar properties [40] but the body of evidence is greatest for theophylline. Though the complete molecular mechanism of the anti-inflammatory properties of theophylline remains unknown it has been shown that theophylline reduces TNF $\alpha$ and IL-1 $\beta$ secretion by monocytes and other immune cells and increases IL-10 release [41]. This is probably mediated through induction of histone deacetylase-dependent gene switching toward a more anti-inflammatory phenotype in immune cells rather than by phosphodiesterase inhibition $[39,42]$. Theophylline also reduces IL-6 levels in peripheral blood [43]. IL-6 however, has a complex role that is dependent on physiological context, such that the sharp transient rise in IL-6 that occurs after exercise appears to have an anti-inflammatory effect, possibly mediated by IL10 release but also directly through an up-rating of insulin receptor sensitivity and an antisarcopenic influence on skeletal myocytes $[44,45]$. On the other hand, the higher resting trough IL-6 levels that are associated with chronic and acute pro-inflammatory states and trauma appear to increase the risk of delirium [17] and are probably pro-sarcopenic [46-49]. There is no evidence regarding the effect of theophylline or similar drugs on post-exercise IL6 . The mechanism of these effects appears to be due to theophylline-induced re-direction toward the anti-inflammatory state via gene switching in macrophages and other immune cells which have been shown to have several dosedependent gene switches that up- and downregulate various cytokines [42,50,51]. An interesting observation is that subjects with COPD treated with the addition of theophylline to a standard regimen had lower baseline CRP levels and better functional scores compared with control subjects [52]. These effects have been described as immune "modulation" by some authors as it appears that theophylline at currently used therapeutic doses tends to reduce inflammation without compromising the protective effect of an appropriate acute inflammatory response to infection, and a recent study has shown a reduction in mortality in patients with severe sepsis treated with theophylline in a critical care setting $[53,54]$.

Theophylline has been shown to cause a small increase in cortisol secretion in normal and asthmatic human subjects at doses used for broncho-dilatation. This effect was transient and tended to revert to baseline after three days, and 
was offset by increased urinary cortisol excretion, and no rise in serum cortisol levels was found [55]. Therefore, it is unlikely that these minor changes in cortico-steroid dynamics would contribute to the anti-inflammatory properties of theophylline.

A potentially beneficial anti-inflammatory effect also occurs at in vitro theophylline concentrations well below the level needed for effective broncho-dilatation [56]. At these lower concentrations $(5-10 \mathrm{mg} / \mathrm{L})$ it is unusual to encounter side effects or signs of theophylline toxicity, such as tachycardia, cardiac arrhythmias, tremor, nausea, diuresis and sleep disturbance. Those side effects are largely mediated by a combination of adenosine receptor blockade and increased release of catecholamines in response to theophyllinestimulated enhancement of intracellular cyclicAMP concentration as a consequence of phosphodiesterase inhibition. That mechanism is a key pathway for broncho-dilatation but is probably not the mechanism for the observed immune modulation effects. At broncho-dilator therapeutic concentration theophylline has been shown to increase the basal metabolic rate (BMR) significantly in laboratory animals, mainly by increasing heart rate, respiratory rate and tremor. At lower anti-inflammatory concentrations, such an effect on BMR is likely to be negligible and of no clinical importance, and no measurable effect was found at therapeutic concentrations in COPD patients at rest [57].

\subsection{Hypothesis}

Based on the evidence presented and cited in the paragraphs above we hypothesize that theophylline at target plasma levels below 10 $\mathrm{mg} / \mathrm{L}$ will down-regulate the pro-inflammatory state and reduce the production of TNF and other pro-inflammatory components, and concomitantly, or consequentially, up-regulate the production of $\mathrm{IL}-10$ and other antiinflammatory bio-chemicals and immune cell phenotypes. We also hypothesize that such an alteration in the overall balance of proinflammatory and anti-inflammatory cytokines will lead to earlier and more complete resolution of the post-infective or post-traumatic systemic inflamed state frequently observed in elderly patients. In other words, a shift from pattern 4 to pattern 3 is predicted (Fig. 2).

We also hypothesize that the predicted shortening of the post-infective, or post- traumatic, inflammatory phase could lead to faster recovery from, or even avoidance of, important neuronal dysfunctions such as delirium, impaired balance, anorexia, low mood, lethargy and autonomic impairment, as well as reducing generalized oedema, myocyte dysfunction, insulin resistance and other adverse metabolic states. Thereby, the treated patient is expected to have a lower risk of becoming overtly cachectic, frail and dependent. In the case of infection, it is probable that the stabilizing effect on innate immune chemistry that our hypothesis predicts will be most beneficial if theophylline treatment is started towards the end of antibiotic treatment so as to cause no blunting of the initial beneficial switch to a protective proinflammatory state as micro-organisms are destroyed, but before the detrimental prolonged tail of non-resolving inflammation is established. The timing for benefit after trauma will probably require an earlier introduction of theophylline, and for elective surgery it is feasible that a preoperative start might be optimal. The ideal duration of treatment will probably be in the 3-6 week range, though further light can only be shed on these practical issues by empirical research.

\subsection{Alternative Immune-modulating Drugs}

We acknowledge that drugs in other classes also influence the innate inflammatory response. Monoclonal antibodies (MCAs) have been used with great success in inflammatory diseases, with anti-TNF drugs for rheumatoid arthritis being the best known example. However, MCAs have the disadvantage of being specific for their target cytokines or cytokine receptors and therefore appear to have a narrower influence on immune settings. They also tend to over-suppress some aspects of immune surveillance and predispose to opportunistic infection and some malignancies, particularly lymphomas, though the latter seems only to occur after prolonged treatment. They are also expensive, have to be given by injection and require close monitoring.

Corticosteroids (CSs) have a long track record of use as broad-spectrum anti-inflammatory drugs that act by down-regulation of pro-inflammatory immune cell behaviour rather than by reestablishing the baseline equilibrium of the innate immune system. When used systemically, they are limited by their mineralocorticoid effects (sodium and water retention), glucocorticoid effects (enhanced protein catabolism and glucose intolerance), tendency to cause increased wakefulness and precipitate delirium, 
and suppression of cell-mediated immunity. Interestingly, there is evidence that the antiinflammatory effects of CSs is augmented by theophylline, including a steroid-sparing effect allowing lower doses and shorter CS courses for exacerbations of COPD [58].

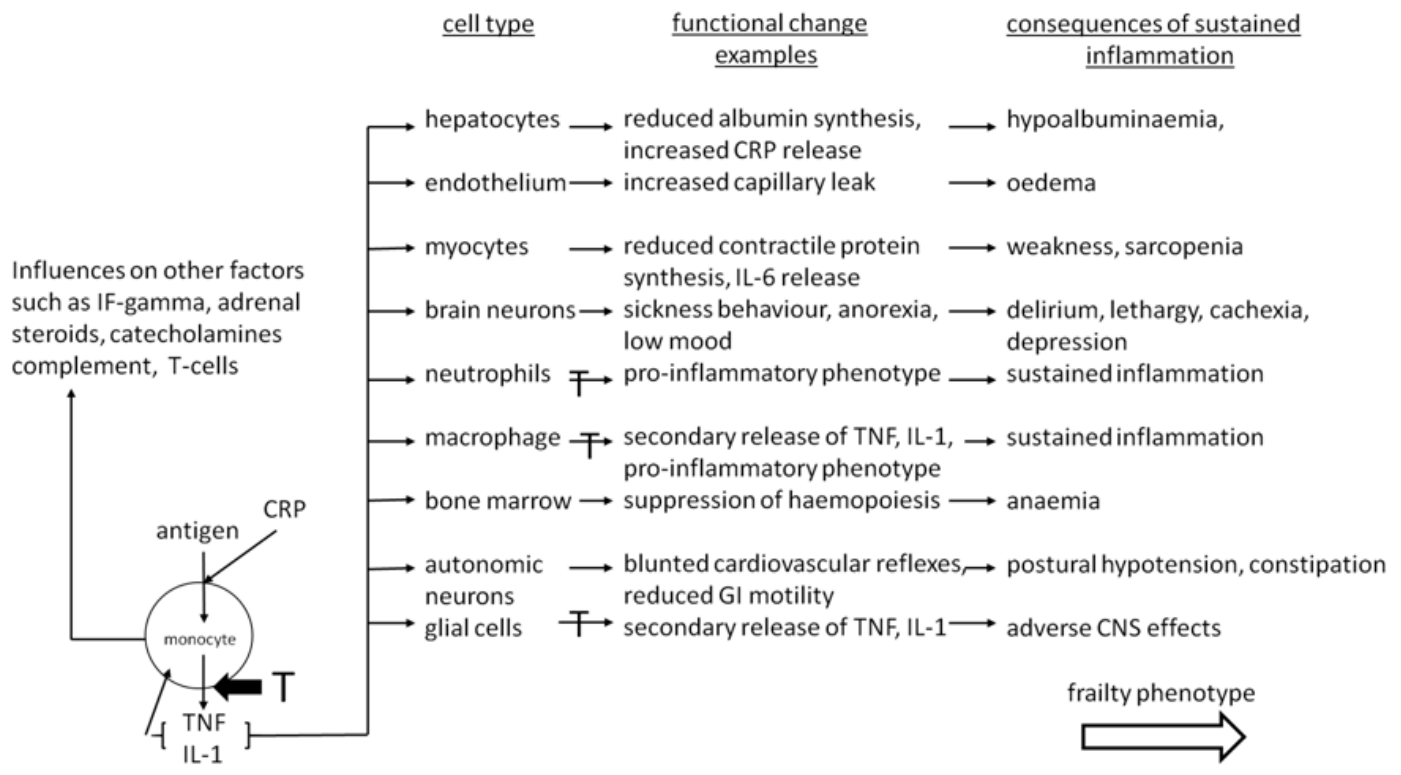

Fig. 1. A simplified schematic representation of TNF and IL-1 release from monocytes in response to an antigen and the subsequent effects on various cells, functional changes and longer term consequences in individuals with prolonged post-acute inflammation (pattern 4). Key: TNF - tumour necrosis factor, IL-1 - interleukin-1, CRP - C-reactive protein, CNS - central nervous system, $T$-probable sites of action of theophylline in its anti-inflammatory role

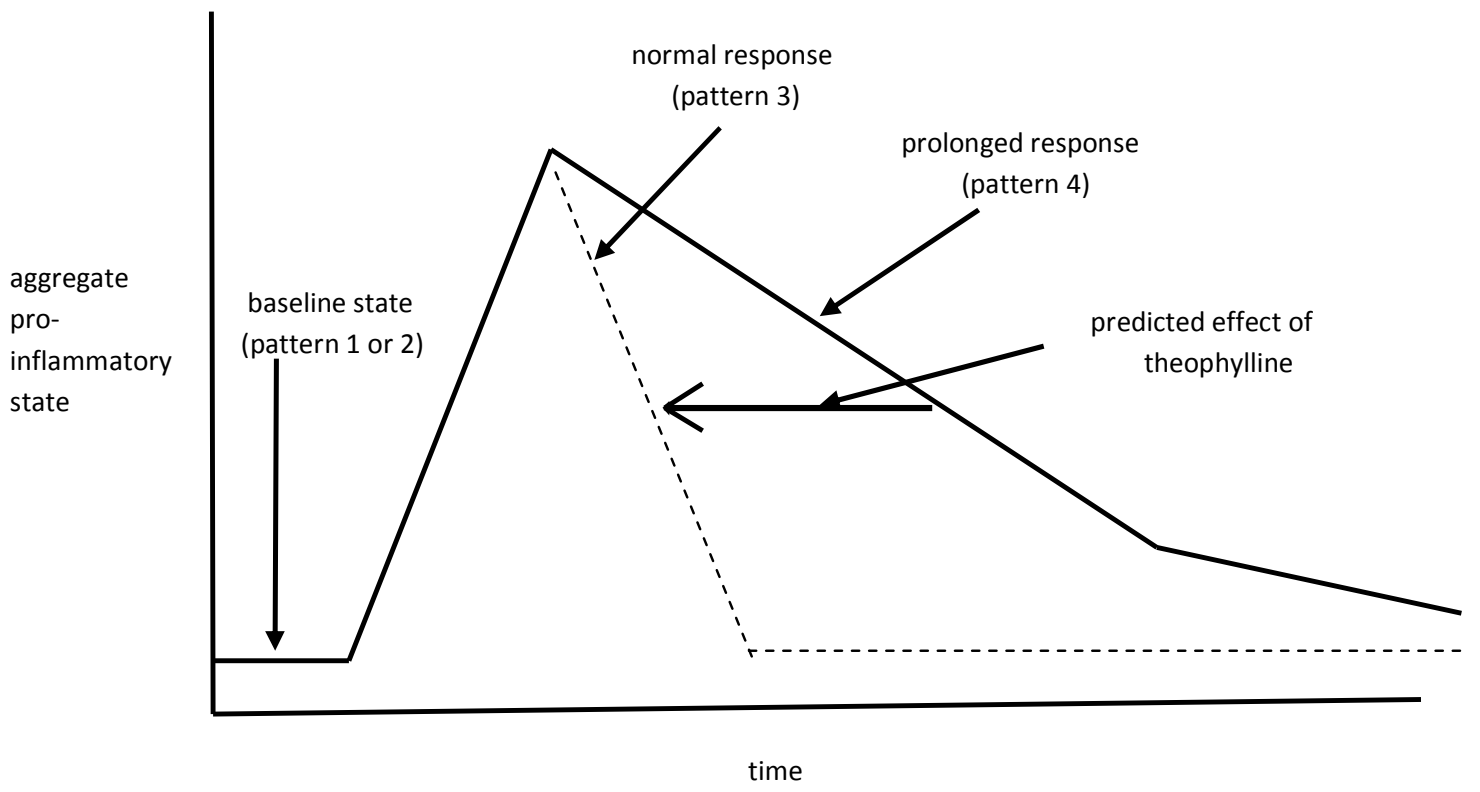

Fig. 2. Diagram to illustrate the hypothesized influence of theophylline on an aggregated proinflammatory state. It is predicted that treatment with theophylline will shift the curve to the left (pattern 4 towards pattern 3 ) and thereby reduce the patient's exposure to an inappropriately prolonged period of inflammation 
Thalidomide and related drugs are established as anti-inflammatory agents with clinical uses in the treatment of leprosy, other mycobacterial diseases, and as an adjunctive drug in certain oncology chemotherapy regimens. Like methylxanthine drugs, thalidomide appears to have subtle immune modulating properties that include suppression of pro-inflammatory cell behaviour, and a reduction of IL-1 $\beta$, IL- 6 and TNF $\alpha$ release $[59,60]$. It is limited by a wide range of sideeffects at therapeutic doses.

4-aminoquinoline drugs (chloroquine, hydroxychloroquine and amodiaquine) have a suppressive action on the release if IL-1 $\beta$, IL-6, TNF $\alpha$ and interferon gamma and tend to promote an increase in the population of immune cells producing IL-10 and IL-4 [61]. They have been used as anti-inflammatory drugs in certain autoimmune disorders such rheumatoid arthritis and systemic lupus erythematosus. The effects of low doses on cytokine dynamics is not known and at usual therapeutic doses a high proportion of patients report unpleasant side effects, though anti-inflammatory doses are usually lower than those used for the treatment of malaria.

Beta-adrenergic receptor blockers, such as propranolol and metoprolol, have been found to alter innate immune responses, with a tendency toward an anti-inflammatory shift. This has largely been observed in relation to the inflammatory profile seen in chronic heart failure and atheromatous vascular disease $[62,63]$. The mechanism is probably by adrenergic receptor blockade and a consequent reduction in catecholamine-mediated release of proinflammatory cytokines by monocytes.

Statins have an anti-inflammatory effect that appears to be independent of, or not directly related to, the induced changes in plasma lipids. The reduction in endothelial inflammation, probably mediated by a protective effect of statins on TNF-induced increases in reactive oxygen species in endothelial cells, has received most attention, but there is evidence of a more systemic effect [64]. However, there have been no studies of the effect of statins on acute-phase inflammation in the context of sepsis and trauma.

\section{DISCUSSION AND SUGGESTED RESEARCH PATHWAYS TO TEST THE HYPOTHESIS}

If our hypothesis proves to be correct, it will offer opportunities to intervene beneficially to reduce the likelihood of progression to overt frailty in elderly patients with acute inflammatory illnesses, including infection, trauma and elective surgery. If low concentrations of theophylline are confirmed as being effective it is likely that side effect symptoms will be minimal, and the treatment therefore acceptable to patients and safe. Of course, adjunctive treatment with theophylline would not replace other aspects of high quality care, such as antibiotics, optimal management of co-morbidities, careful fluid and electrolyte management and prophylactic anticoagulation, all of which can contribute to improving clinical and functional outcomes.

It will be necessary to take a somewhat more reductive and focussed approach to test the hypothesis. Exploration of the mechanisms, beginning with identifying the key steps in the chain of cause and effect will need to be based initially on animal studies, and in the case of delirium will probably need to define more precisely the effect of theophylline on IL-1, IL-6 and TNF-induced changes in the function of neurons, glial cells and blood-brain barrier permeability. There are a number of animal models of acute inflammation that can be used to quantify the effect of theophylline on cytokine responses, behaviour patterns, muscle morphology and function.

We argue that it is not essential to try to establish a complete understanding of such mechanisms before embarking on a clinical intervention trial in humans; the full range of mechanisms of action of very few drugs have been completely defined, and it might not be possible to achieve such a full definition in the innate immunity field due to immune-regulatory complexity and individual variation. However, the body of evidence that underpins our hypothesis is sufficiently robust to justify a trial of theophylline in humans. The most controllable setting is probably elective surgery, which obviously allows the pre-traumatic inflammatory state to be measured, and therefore sets a target baseline identical to either pattern 1 or pattern 2 for elderly subjects. A controlled model for the effects of sepsis could be conducted in volunteers given a standardized dose of a bacterial-derived antigen such as enterotoxin, which would also facilitate pre- and post-exposure measurements to be made. It will be important at a later stage to study patients with acute infections, perhaps using pneumonia or uro-sepsis as the initiating stimuli, because these represent a large proportion of cases seen in clinical practice. Of course, in such subjects 
the pre-infection baseline state will not be known. In all these empirical frameworks, we envisage a randomized placebo-controlled trial of theophylline at a target plasma concentration of $5-10 \mathrm{mg} / \mathrm{L}$ with measurements of key plasma cytokines (for example IL-1 1 , TNF $\alpha$, IL-6, IL-10, IL-1ra), other chemokines involved in inflammation, serum CRP and albumin, body weight, hand-grip, standardized walking performance, functional scores (for example Barthel Index), psychometric tests (for delirium, depression, cognitive impairment), length of stay in hospital, discharge care needs, 30-day all-cause mortality, drug side-effects, proportion of trial completers and rate of readmission to hospital. Further exploration of the concept would depend on the outcomes of such initial trials.

\section{CONCLUSION}

There is a growing understanding of the part played by systemic inflammation in the genesis of frailty in older people, including the progression to sarcopenia. It is also increasingly clear that disordered control of the innate immune system is a key factor in the pathophysiological pathway leading to several aspects of the frailty syndrome at all ages, but with most of the clinical burden falling on elderly patients with chronic inflammatory conditions, or delayed resolution of acute inflammation. In such patients, in addition to attention to nutrition and moderate exercise, there is theoretical, laboratory, observational and some clinical evidence that a number of drugs with immune modulating properties could be used to reduce the pro-inflammatory state, with potential benefit to wellbeing, physiological performance and independent function. Theophylline is arguably one of the most attractive candidates for use in an anti-inflammatory role because it appears to retain its immune-modulating properties at plasma concentrations well below the toxic range. Properly conducted clinical trials are needed to establish the place of theophylline in this clinical context.

\section{CONSENT}

It is not applicable.

\section{ETHICAL APPROVAL}

It is not applicable.

\section{COMPETING INTERESTS}

Authors have declared that no competing interests exist.

\section{REFERENCES}

1. Yeh SS, Schuster MW. Geriatric cachexia: The role of cytokines. Am J Clin Nutr. 1999;70:183-97.

2. Francheschi C, Campisi J. Chronic inflammation (inflammaging) and its potential contribution to age-associated diseases. J Gerontol A Biol Sci Med Sci. 2014;69(S1):S4-S9.

3. Allen SC. Ageing, exercise and the chemistry of inflammation. Rev Clin Gerontol. 2015;25:73-80.

4. Casperan CJ, Pereira MA, Curran KM. Changes in physical activity patterns in the United States, by sex and cross-sectional age. Med Sci Sports Exerc. 2000;32: 1601-9.

5. Driver JA, Djousse L, Logroscino G, Gaziano JM, Kurth $T$. Incidence of cardiovascular disease and cancer in advanced age: Prospective cohort study. BMJ. 2008;337:a2467.

6. Chung HY, Cesari M, Anton S, et al. Molecular inflammation: Underpinnings of aging and age-related diseases. Ageing Res Rev. 2009;8:18-30.

7. Pedersen BK. The anti-inflammatory effect of exercise: Its role in diabetes and cardiovascular disease control. Essays Biochem. 2006;42:105-17.

8. Bruunsgaard H, Pedersen BK. Age-related inflammatory cytokines and disease. Immunol Allergy Clin North Am. 2003;23: 15-39.

9. Bruunsgaard $H$, Skinhoj $P$, Qvist J, Pedersen BK. Elderly humans show prolonged in vivo inflammatory activity during pneumococcal infections. J Infect Dis. 1999;180:551-4.

10. Krabbe KS, Bruunsgaard $\mathrm{H}$, Hansen CM, et al. Ageing is associated with a prolonged fever response in human endotoxemia. Clin Diagn Lab Immunol. 2001;8:333-8.

11. Jaffer U, Wade RG, Gourlay T. Cytokines in the systemic inflammatory response syndrome. HSR Proc Intensive Care Cardiovasc Anesth. 2010;2:161-75.

12. Hoffman J, Ahira S. Innate immunity. Curr Opin Immunol. 2013;25:1-3. 
13. DeForge LE, Remick DG. Kinetics of TNF, IL-6 and IL-8 gene expression in LPSstimulated human whole blood. Biochem Biophys Res Comm. 1991;174;18-24.

14. Jansky L, Reymanova P, Kopecky J. Dynamics of cytokine production in human peripheral blood mononuclear cells stimulated by LPS or infected by Borrelia. Physiol Res. 2003;52:593-8.

15. Shaw AC, Joshi S, Greenwood H, Panda A. Aging of the innate immune system. Corr Opin Immunol. 2010;22:507-13.

16. Poon DCH, Ho YS, Chiu K, Chang CC. Cytokines: How important are they in mediating sickness? Neurosci Biobehav Rev. 2013;37:1-10.

17. Vasunilashorn SM, Ngo L, Inouye SK, et al. Cytokines and postoperative delirium in older patients undergoing major elective surgery. J Gerontol A Biol Sci Med Sci. 2015;70:1289-95.

18. Serhan CN, Brain SD, Buckley CD, et al. Resolution of inflammation: State of the art, definitions and terms. J Fed Amer Soc Exper Biol. 2007;21:32532.

19. Castle SD. Clinical relevance of agerelated immune dysfunction. Clin Infect Dis. 2000;31:578-85.

20. Opal SM, Girard TD, Ely WE. The immunopathogenesis of sepsis in elderly patients. Clin Infect Dis. 2005;41(supp 7): S504-S512.

21. Woods JA, Wilund KR, Martin SA, Kistler BM. Exercise, inflammation and aging. Aging Dis. 2012;3:130-40.

22. Cowie CC, Rust KF, Byrd-Holt DD, et al. Prevalence of diabetes and impaired fasting glucose in adults in the US population: NHANES survey 1999-2002. Diabetes Care. 2006;29:1263-8.

23. Kalaria RN, Maestre GE, Arizaga R, et al. Alzheimer's disease and vascular dementia in developing countries: Prevalence, management and risk factors. Lancet Neurol. 2008;7:812-26.

24. Coresh J, Selvin E, Stevens LA, et al. Prevalence of chronic kidney disease in the United States. JAMA. 2007;298:203847.

25. Dagenais S, Garbedian S, Wai EK. Systematic review of the prevalence of radiographic primary hip osteoarthritis. Clin Orthop Relat Res. 2009;467:623-37.
26. Christian LM, Glaser R, Porter K, Malarkey WB, Beversdorf D, Kiecolt-Glaser JK. Poorer self-related health is associated with elevated inflammatory markers among older adults. Psychoneuroendocrinology. 2011;36:1495-504.

27. Michaud M, Balardy L, Moulis G, et al. Proinflammatory cytokines, aging, and age-related diseases. J Amer Med Dir Assoc. 2013;14:877-82.

28. Tousoulis D, Charakida M, Stefanadis C. Endothelial function and inflammation in coronary artery disease. Heart. 2006;92: 441-4.

29. Dantzer R. Cytokine, sickness behaviour, and depression. Immunol Allergy Clin North Am. 2009;29:247-64.

30. Banks WA, Kastin AJ, Broadwell RD. Passage of cytokines across the bloodbrain barrier. Neuroimmunomodulation. 1995;2:241-8.

31. Banks WA. Blood-brain barrier transport of cytokines. Neuroimmune Biol. 2008;6: 93-107.

32. Yarlagadda A, Alfson E, Clayton AH. The blood-brain barrier and the role of cytokines in neuropsychiatry. Psychiatry (Edgmont). 2009;6:18-22.

33. Pan W, Stone KP, Hsuchou H, Manda VK, Kastin AJ. Cytokine signalling modulates blood-brain barrier function. Curr Pharm Des. 2011;17:3729-40.

34. Tizard I. Sickness behaviour, its mechanisms and significance. Anim Health Res Rev. 2008;9:87-99.

35. Srinivasan D, Yen JH, Joseph DJ, Friedman W. Cell type-specific interleukin1 beta signalling in the CNS. J Neurosci. 2004;24:6482-8.

36. Carlson NG, Whitney WA, Chen J, Bacchi A, Rogers SW, Gahring LC. Inflammatory cytokines IL-1 alpha, IL-1 beta, IL-6 and TNF alpha impart neuroprotection to an excitotoxin through distinct pathways. J Immunol. 1999;163:3963-8.

37. Oikonomopoulou K, Ricklin D, Ward PA, Lambris JD. Interactions between coagulation and complement: Their role in inflammation. Semin Immunopathol 2012; 34:151-65.

38. Barnes PJ. Theophylline for COPD. Thorax. 2006;61:742-4.

39. Barnes PJ. Theophylline in COPD. Proc Amer Thorac Soc. 2005;2:334-9. 
40. Neuner P, Klosner G, Schauer E, et al. Pentoxyfylline in vivo down-regulates the release of IL-1 beta, IL-6, IL- 8 and TNF alpha by human peripheral blood mononuclear cells. Immunology. 1994;83: 262-7.

41. Cvietusa P, Mascali JJ, Negri J, Borish L. Anti-inflammatory effects of theophylline: Modulation of cytokine production. Ann Allergy Asthma Immunol. 1996;77:34-8.

42. Ito K, Lim S, Caramori G, et al. A molecular mechanism of the action of theophylline: Induction of histone deacetylase activity to decrease inflammatory gene expression. Proc Nat Acad Sci. 2002;99:8921-6.

43. Ichiyami $\mathrm{T}$, Hasegawa $\mathrm{S}$, Matsubara $\mathrm{T}$, Hayashi T, Furukawa S. Theophylline inhibits NF-kappa activation and I kappa B alpha degradation in human pulmonary epithelial cells. Arch Pharmacol. 2001;364: 558-61.

44. Pal M, Febbraio MA, Whitham M. From cytokine to myokine: The emerging role of IL-6 in metabolic regulation. Immunol Cell Biol. 2014;92:331-39.

45. Pedersen BK, Febbraio M. Muscle-derived IL-6: A possible link between skeletal muscle, adipose tissue, liver and brain. Brain Behav Immun. 2005;19:371-6.

46. Mikkelsen UR, Couppe C, Karlsen A, et al. Life-long endurance exercise in humans: Markers and leg muscle size. Mech Ageing Dev. 2013;134:531-40.

47. Woods JA, Veira VJ, Keylock KT. Exercise, inflammation and innate immunity. Immunol Allergy Rev. 2006;12: 6-33.

48. Pedersen BK. Exercise-induced myokines and their role in chronic disease. Brain Behav Immun. 2011;25:811-6.

49. Brandt C, Pedersen BK. The role of exercise-induced myokines in muscle homeostasis and the defence against chronic diseases. J Biomed Biotechnol; 2010.

Available:http://dx.doi.org/10.1155/2010/52 $\underline{0258}$

(Accessed 3 January 2015).

50. Spatafora M, Chiappara G, Merendino AM, D'Amico D, Bellia V, Bonsignore G. Theophylline suppresses the release of TNF alpha by blood monocytes and alveolar macrophages. Eur Respir J. 1994; 7:223-8.
51. Yoshimura T, Usami E, Kurita C, et al. Effect of theophylline on the production of IL-1 beta, TNF alpha and IL-8 by human peripheral blood mononuclear cells. Biol Pharm Bull. 1995;18:1405-8.

52. Subramanian $V$, Ragulan $A B$, Jindal $A$, Wiswambhar $V$. The study of tolerability and safety of theophylline given along with formoterol plus budesonide in COPD. J Clin Diagn Res. 2015;9:10-3.

53. Hancock REW, Nijnik A, Philpott DJ. Modulating immunity as a therapy for bacterial infections. Nature Rev Microbiol. 2012;10:243-54.

54. Shih YN, Chen YT, Seethala R, Aisiko I, Frendl G, Hou P. Effect of the use of theophylline and sepsis outcomes. Crit Care Med. 2015;43:274.

55. Tulin-Silver J, Schteingart DE, Mathews KP. Effect of theophylline on cortisol secretion. J Allergy Clin Immunol 1981;67: 45-50.

56. Zhang J, Feng MX, Qu JM. Low dose theophylline showed an inhibitory effect on the production of IL-6 and IL-8 in primary lung fibroblasts from patients with COPD. Mediators Inflamm; 2012.

DOI: $10.1155 / 2012 / 492901$

(Accessed June 19 2016)

57. Mosire K, Renvall MJ, Ramsdell JW, Spindler AA. The effect of theophylline on metabolic rate in COPD patients. J Am Coll Nutr. 1966;15:403-7.

58. Cosio BG, Iglesias A, Rios A, et al. Lowdose theophylline enhances the antiinflammatory effects of steroids during exacerbations of COPD. Thorax. 2009;64: 424-9.

59. Bodera P, Stankiewicz W. Immunomodulatory properties of thalidomide analogs: Pomalidomide and lenalidomide, experimental and therapeutic applications. Recent Pat Endocr Metab Immune Drug Discov. 2011;5:192-6.

60. Eski M, Sahin I, Sengezer M, Serdar M, Ifran $\mathrm{A}$. Thalidomide decreases the plasma levels of IL-1 and TNF following burn injury: Is it the new drug for modulation of systemic inflammatory response. Burns. 2008;34:104-8.

61. van den Borne BE, Dijkmans BA, de Rooij $\mathrm{HH}$, le Cessie S, Verweij CL. Chloroquine and hydroxychloroquine equally affect TNF alpha, IL-6 and IF gamma production by 
peripheral blood mononuclear cells. J Rheumatol. 1997;24:55-60.

62. Ohtsuka T, Hamada M, Hiasa G, et al. Effect of beta-blockers on circulating levels of inflammatory and anti-inflammatory cytokines in patients with dilated cardiomyopathy. J Am Coll Cardiol. 2001; $37: 412-7$

63. Ulleryd MA, Bernberg E, Yang LJ, Bergstrom GML, Johansson ME.

Metoprolol reduces pro-inflammatory cytokines and atherosclerosis in ApoE-/Mice. Biomed Res Internat; 2014.

DOI: $10.1155 / 2014 / 548783$

(Accessed June 10 2016)

64. Wu K, Tian S, Zhou H, Wu Y. Statins protect human endothelial cells from TNF-induced inflammation via ERK5 activation. Biochem Pharmacol. 2013;85:1753-60.

(c) 2016 Allen et al.; This is an Open Access article distributed under the terms of the Creative Commons Attribution License (http://creativecommons.org/licenses/by/4.0), which permits unrestricted use, distribution, and reproduction in any medium, provided the original work is properly cited.

Peer-review history:

The peer review history for this paper can be accessed here: http://sciencedomain.org/review-history/17912 